\title{
The "plastic healing concept": implantation of bioabsorbable scaffolds in spontaneous coronary artery dissection
}

\author{
Ahmed Al Mamary, MD; Gilberto Dariol, MD; Massimo Napodano*, MD \\ Cardiac Catheterization Laboratories and Interventional Cardiology, Department of Cardiac, Thoracic and Vascular Sciences, \\ University of Padua, Padua, Italy
}

This paper also includes accompanying supplementary data published online at: http://www.pcronline.com/eurointervention/82nd_issue/225

A 45-year-old woman presented to our department because of inferior STEMI. A coronary angiogram showed patent coronary arteries with a near-occlusive smooth lesion from the mid to distal large obtuse marginal (OM), but otherwise normal vessels (Figure 1A). IVUS indicated the presence of intramural haematoma, with thrombosis of false lumen compressing the true lumen (Figure 1B, Moving image 1). Two bioabsorbable scaffolds (Absorb $3.5 \times 12 \mathrm{~mm}+3.0 \times 18 \mathrm{~mm}$; Abbott Vascular, Santa Clara, CA, USA) were directly deployed with short overlap. Angiogram/IVUS revealed complete scaffold deployment, with fully apposed struts to the intimomedial membrane and no residual haematoma outside the scaffold area (Figure 1C, Figure 1D, Moving image 2). Six months later, a coronary computed tomography angiogram revealed the patency of the BVS with no residual haematoma of the vessel wall outside the scaffold area (Figure 1E, Figure 1F).

\section{Conflict of interest statement}

The authors have no conflicts of interest to declare.

\section{Online data supplement}

Moving image 1. Intravascular ultrasound scan shows the presence of false lumen (9 o'clock to 2 o'clock) of the obtuse marginal branch with partially thrombosed haematoma, compressing the true lumen, without clear imaging of intimal tear appreciable.

Moving image 2. Intravascular ultrasound of the obtuse marginal branch after the implantation of two bioabsorbable vascular scaffolds, showing uniform full expansion of scaffolds apposed to the intima, with healing of abluminal haematoma.
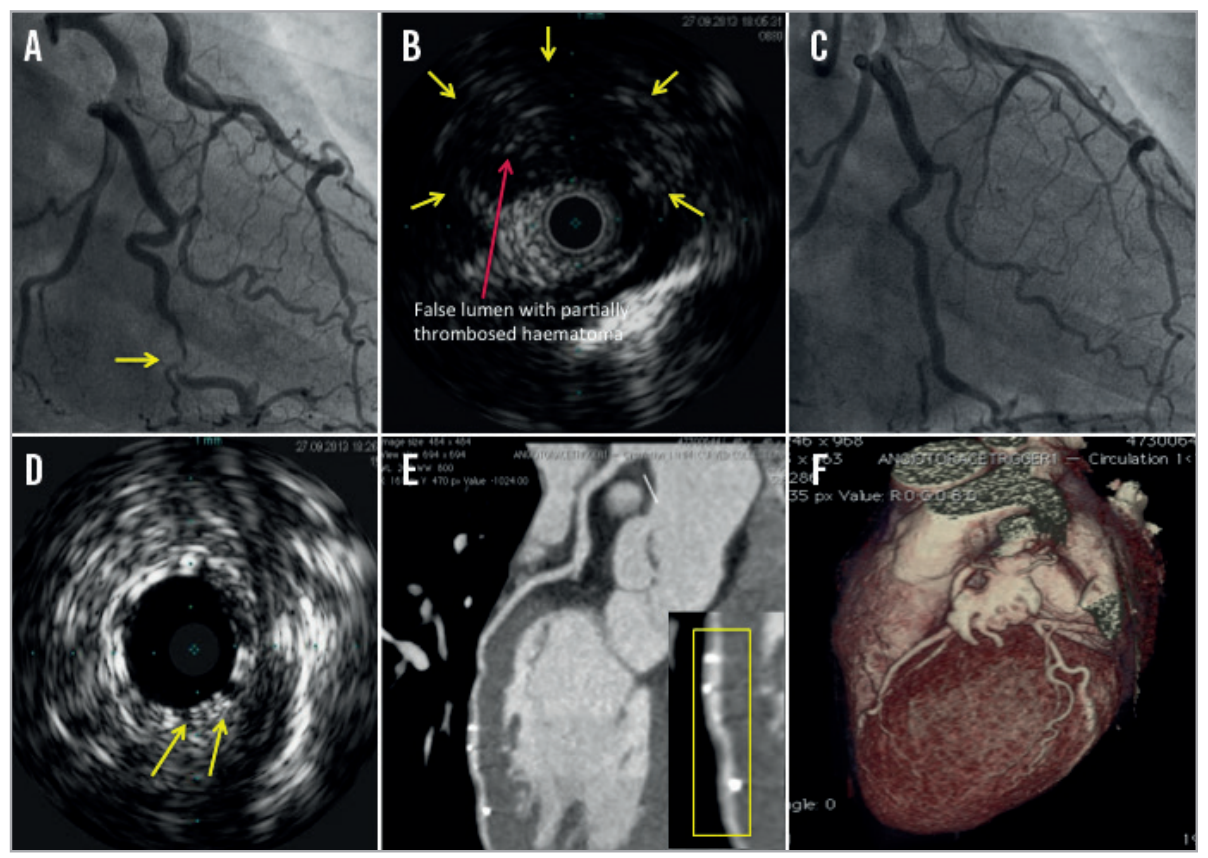

Figure 1. Imaging of SCAD before and after BVS implantation. A) Angiogram showing smooth lesion of the obtuse marginal (OM) (yellow arrowhead). B) IVUS shows false lumen (yellow arrowheads), with partially thrombosed haematoma, compressing the true lumen (red arrowhead). C) \& D) Angiogram/IVUS after two bioabsorbable vascular scaffolds (BVS) had been implanted, showing full expansion of the scaffolds apposed to the intimomedial membrane. E) \& F) Six-month computed tomography angiogram reveals the patency of the BVS (yellow rectangle), with no residual haematoma of the vessel wall outside the scaffold area.

*Corresponding author: Cardiac Catheterization Laboratories and Interventional Cardiology, Department of Cardiac, Thoracic and Vascular Sciences, University of Padua, 2 Via Giustiniani, 35100 Padua, Italy.E-mail: massimo.napodano@gmail.com 